\title{
Melanoma is associated with an increased risk of bullous pemphigoid: a large population-based longitudinal study
}

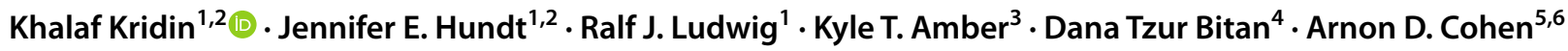

Received: 2 December 2020 / Revised: 6 February 2021 / Accepted: 13 February 2021 / Published online: 9 March 2021

(c) The Author(s) 2021

\begin{abstract}
The association between bullous pemphigoid (BP) and melanoma is yet to be investigated. We aimed to assess assess the bidirectional association between BP and melanoma and to delineate the epidemiological features of patients with both diagnoses. A population-based cohort study was performed comparing BP patients $(n=3924)$ with age-, sex- and ethnicitymatched control subjects $(n=19,280)$ with regard to incident cases of melanoma. A case-control design was additionally adopted to estimate the risk of BP in individuals with a preexisting diagnosis of melanoma. The prevalence of preexisting melanoma was higher in patients with BP than in control subjects ( $1.5 \%$ vs. $1.0 \%$, respectively; $P=0.004)$. A history of melanoma confers a $50 \%$ increase in the risk of subsequent BP (OR 1.53; 95\% CI 1.14-2.06). This risk was higher among males (OR 1.66; 95\% CI 1.09-2.54) and individuals older than 80 years (OR 1.63; 95\% CI 1.11-2.38), and persisted after adjustment for multiple putative confounders including PD-1/PDL-1 antagonists (adjusted OR 1.53; 95\% CI 1.14-2.06). Conversely, the risk of melanoma among patients with BP was slightly elevated, but did not reach the level of statistical significance (adjusted HR 1.13; 95\% CI 0.73-1.74). Patients with a dual diagnosis of BP and melanoma were older at the onset of BP and had lower body mass index. A history of melanoma is associated with a $50 \%$ increase in the incidence of subsequent BP. Physicians managing patients with both conditions should be aware of this association. Further research is warranted to reveal the underlying mechanism of these findings.
\end{abstract}

\section{Introduction}

Bullous pemphigoid (BP) is the most prevalent subepidermal autoimmune bullous disease worldwide [1]. The disease is characterized by the presence of circulating $\operatorname{IgG}$

Khalaf Kridin and Jennifer E. Hundt equal contribution.

Khalaf Kridin

dr_kridin@hotmail.com

1 Lübeck Institute of Experimental Dermatology, University of Lübeck, Ratzeburger Allee 160, 23562 Lübeck, Germany

2 Azrieli Faculty of Medicine, Bar-Ilan University, Safed, Israel

3 Department of Dermatology, University of Illinois at Chicago, Chicago, IL, USA

4 Department of Behavioral Sciences, Ariel University, Ariel, Israel

5 Clalit Health Services, Tel-Aviv, Israel

6 Siaal Research Center for Family Medicine and Primary Care, Faculty of Health Sciences, Ben-Gurion University of the Negev, Beer Sheva, Israel autoantibodies against BP180 and BP230, hemidesmosomal proteins promoting dermal-epidermal cohesion [2,3]. Binding of these autoantibodies initiates blister formation via dermal-epidermal separation, which has been shown to be directly pathogenic [4]. The disease is clinically characterized by tense bullae, often in conjunction with urticarial plaques, and characteristically affects elderly individuals aged over 70 years. The last decades witnessed a considerable increase in the risk of the disease, which has been attributed, at least in part, to increased $f$ longevity and exposure to several drugs [5]. The past few years yielded a prominent advance in elucidating the comorbidity profile of patients with BP owing to an increasing number of well-designed controlled observational studies $[1,6]$.

Melanoma is a malignant tumor that arises from melanocytes and is typified by a strong capacity to metastasize. While most commonly cutaneous in origin, it can potentially develop in mucosal surfaces, within the uveal tract, or in the leptomeninges [7]. Owing to its metastatic potential, melanoma accounts for more than $90 \%$ of skin cancer-related mortality [8]. The incidence of melanoma has risen substantially over the past four decades, whilst mortality rates 
follow a stable course since the early 1990s [9]. The prognosis of patients with melanoma varies in accordance with the stage at diagnosis. While patients with localized primary cutaneous melanoma have a favorable prognosis, the median survival time of patients with distant metastasis (before the advent of immunotherapies) was estimated at 9 months [7].

The coexistence of BP and melanoma was described in several case reports, but the association between these conditions is yet to be investigated by controlled observational studies [10]. The presence of a putative association between $\mathrm{BP}$ and melanoma was also postulated in view of several experimental and serological findings like the expression of BP180 in malignant melanocytes [11], the progression of melanoma in a mouse strain with BP180 dysfunction [12], and the increased seropositivity of anti-BP230 autoantibodies among melanoma patients [13].

The aim of the current study is to evaluate the association between BP and melanoma using a large-scale populationbased study. We additionally aimed to examine whether patients with BP and melanoma possess a unique epidemiological profile relative to the remaining patients with BP.

\section{Methods}

\section{Study design and dataset}

The current study was performed to investigate the bidirectional association between BP and melanoma using one of the large cohorts of patients with BP in the literature. To delineate the risk of developing melanoma during the course of BP, a cohort study design was adopted to follow patients with BP and estimate the incidence of melanoma. To evaluate the risk of having BP in subjects with a preceding history of melanoma, a case-control study design was followed to unveil the prevalence of preexisting melanoma (exposure) in patients with subsequent BP (outcome). Given that 'The rare disease assumption' hypothesizes that estimates produced by case-control studies of rare disease approach that produced by cohort studies [14], the case-control study design allows us to assess the risk of BP in individuals with a history of melanoma.

The computerized dataset of Clalit Health Services (CHS) was the origin of the current study. CHS is the main healthcare maintenance organization in Israel, providing a wide array of private and public healthcare services for 4,927,000 enrollees as of October 2018. CHS retrieves data from a multitude of sources covering all strata of the healthcare system, including primary care healthcare facilities, referral centers settings, laboratory analyses, and imaging data. The loss to follow-up is negligible, and access to CHS services is free, thus increasing its compatibility to give rise to reliable epidemiological studies [15].

\section{Study population and definition of main variables}

The dataset of CHS was systematically checked for incident cases with a diagnostic code BP between the years 2002 and 2019. Eventual eligibility in the study was only fulfilled when a patient met one of the following: (1) a documented diagnosis of BP registered at least twice by a board-certified dermatologist, or (2) a diagnosis of BP in discharge letters of patients admitted to dermatological wards. The diagnosis of melanoma was based on its documentation in the chronic registry of the CHS, which is cross-linked with the Israel National Cancer Registry. The control group was comprised of up to five individuals per each case of BP. Controls were matched based on sex, age, and ethnicity.

\section{Covariates and sensitivity analyses}

To substantiate the validity of our findings, we performed two sensitivity analyses alongside the general analysis; (1) to increase the validity of the diagnosis of BP, only patients who claimed BP-related medications were included. The latter include systemic or topical corticosteroids for more than 6 months, as well as one of the adjuvant immunosuppressive or immunomodulatory agents (azathioprine, mycophenolate mofetil, methotrexate, cyclophosphamide, dapsone, doxycycline, rituximab, plasmapheresis, intravenous immunoglobulins); (2) to refute the presence of major ascertainment bias in the case-control study, we omitted cases of melanoma diagnosed up to 2 years prior to the study commencement.

Given the recent evidence connecting between the use of programmed death (PD)-1/programmed death ligand (PDL)-1 inhibitors in patients with metastatic melanoma and the development of BP [16], the outcome measures were additionally adjusted for this exposure. Since Parkinson disease was found to associate with both BP [17] and melanoma [18], we additionally adjusted for this variable as a putative confounder. To rule out ascertainment bias stemming from overutilization of healthcare services, and to ascertain that the study findings did not merely arise from over diagnosis of melanoma or BP, we additionally adjusted for utilization of healthcare services. This variable was defined as the number of visits to community physicians throughout the study duration (between 2002 and 2019).

\section{Statistical analysis}

Baseline characteristics were described by means and standard deviations (SD)s for continuous variables, whilst categorical values were indicated by percentages. The comparison of different variables between cases and controls was 
performed using the chi-square test and $t$ test for categorical and continuous variables, respectively.

In the cohort study design, incidence rates of melanoma were calculated for both BP patients and controls and expressed as the number of events per 1000 person-years. Hazard ratios (HR)s for the risk of incident melanoma were obtained by the use of the Cox regression model. In the case-control study design, logistic regression was used to calculate odds ratios (ORs) and 95\% confidence intervals (CI)s to compare cases and controls with respect to the presence of preceding melanoma. The association was calculated based on individuals who developed BP after the diagnosis of melanoma, given that a temporal relationship exists between exposure and outcome in case-control studies. In the last section aiming to evaluate the epidemiological and clinical characteristics of patients with BP and melanoma relative to those with isolated $\mathrm{BP}$, all patients with both diagnoses were included regardless of the sequence of their appearance. Two-tailed $P$ values less than 0.05 were considered as statistically significant. All statistical analyses were performed using SPSS software, version 25 (SPSS, Armonk, NY: IBM Corp).

\section{Results}

\section{Characteristics of the study population}

A total of 3924 patients with BP and 19,280 age-, sex, and ethnicity-matched control subjects were included in the current study. The mean (SD) age at the onset of BP was 76.7 (14.3) years, $2257(57.5 \%)$ were females, and 3752 (95.6\%) were of Jewish ancestry. The basal characteristics of the study participants are outlined in Table 1.

\section{The risk of developing BP with a preceding diagnosis of melanoma}

The prevalence rate of preexisting melanoma was greater in patients with BP than in controls $(1.5 \%$ vs. $1.0 \%$, respectively; $P=0.004)$. To elaborate, a 1.5 -fold increase in the risk of BP emerged in those with a history of melanoma (OR 1.53; 95\% CI 1.14-2.06). In a stratified analyses by age, sex, and ethnicity, melanoma was observed to predict the diagnosis of BP in patients older 80 years of age (OR 1.63; 95\% CI 1.11-2.38), males (OR 1.66; 95\% CI 1.09-2.54), and individuals of Jewish ethnicity (OR 1.54; 95\% CI 1.15-2.07; Table 2).

We then performed two sensitivity analyses. The first of which by including only cases managed by BP-related medications and the second omitted cases and controls given a diagnosis of melanoma in the initial 2 years of the study. The association kept its statistical significance in both of these sensitivity analyses (OR 1.54; 95\% CI 1.12-2.11 and OR 1.43 ; $95 \%$ CI 1.06-2.92, respectively).

The association was not meaningfully altered after adjusting for demographic variables (model 1; OR 1.52; 95\% CI 1.13-2.05) as well as for demographic variables alongside comorbidities (model 2; OR 1.54; 95\% CI 1.14-2.08) and additionally for exposure to PD-1/PDL-1 inhibitors, Parkinson's disease, and overutilization of healthcare services (model 3; OR 1.45; 95\% CI 1.07-1.96; Supplementary Table 1).
Table 1 Descriptive characteristics of the study population

\begin{tabular}{|c|c|c|c|}
\hline Characteristic & $\begin{array}{l}\text { Patients with bullous pem- } \\
\text { phigoid }(N=3924)\end{array}$ & Controls $(N=19,280)$ & $P$ value \\
\hline \multicolumn{4}{|l|}{ Age, years } \\
\hline Mean (SD) & $76.7(14.3)$ & $76.3(14.3)$ & 0.904 \\
\hline Median (range) & $79.9(0.4-104.4)$ & $79.5(0.7-103.8)$ & \\
\hline Male sex, $N(\%)$ & $1667(42.5 \%)$ & $8168(42.4 \%)$ & 0.908 \\
\hline \multicolumn{4}{|l|}{ Ethnicity, $N(\%)$} \\
\hline Jews & $3752(95.6 \%)$ & $18,397(95.4 \%)$ & 0.584 \\
\hline Arabs & $171(4.4 \%)$ & $868(4.5 \%)$ & \\
\hline \multicolumn{4}{|l|}{ BMI, $\mathrm{mg} / \mathrm{kg}^{2}$} \\
\hline Mean (SD) & $27.9(6.1)$ & $27.9(8.4)$ & 1.000 \\
\hline Smoking, $N(\%)$ & $1148(29.3 \%)$ & $5771(29.9 \%)$ & 0.454 \\
\hline \multicolumn{4}{|c|}{ Charlson comorbidity score } \\
\hline Mean score (SD) & $3.4(2.4)$ & $2.9(2.3)$ & $<0.001$ \\
\hline None $(0)$ & $468(11.9 \%)$ & $3376(17.5 \%)$ & $<0.001$ \\
\hline Moderate YY (1-2) & $1113(28.4 \%)$ & $6177(32.0 \%)$ & $<0.001$ \\
\hline Severe $(\geq 3)$ & $2343(59.7 \%)$ & $9727(50.5 \%)$ & $<0.001$ \\
\hline
\end{tabular}

$B P$ bullous pemphigoid, $N$ number, $S D$ standard deviation, $B M I$ body mass index 
Table 2 The risk of bullous pemphigoid in patients with a preceding diagnosis of melanoma stratified by age, sex, and ethnicity (case-control study design)

\begin{tabular}{|c|c|c|c|c|}
\hline Subgroup & $\begin{array}{l}\text { Melanoma in patients } \\
\text { with BP } \\
n(\%)^{\mathrm{a}}\end{array}$ & $\begin{array}{l}\text { Melanoma in controls } \\
n(\%)^{\mathrm{a}}\end{array}$ & OR $(95 \%$ CI $)$ & $\begin{array}{l}\text { Univariate } \\
P \text { value }\end{array}$ \\
\hline All & $59(1.5 \%)$ & $190(1.0 \%)$ & $1.53(1.14-2.06)$ & 0.004 \\
\hline \multicolumn{5}{|l|}{ Age, years } \\
\hline$<70$ & $6(0.7 \%)$ & $33(0.7 \%)$ & $0.93(0.39-2.23)$ & 0.873 \\
\hline $71-80$ & $17(1.5 \%)$ & $51(0.9 \%)$ & $1.69(0.97-2.93)$ & 0.061 \\
\hline$\geq 80$ & $36(1.9 \%)$ & $106(1.2 \%)$ & 1.63 (1.11-2.38) & 0.012 \\
\hline \multicolumn{5}{|l|}{ Sex } \\
\hline Male & $29(1.8 \%)$ & $86(1.1 \%)$ & $1.66(1.09-2.54)$ & 0.018 \\
\hline Female & $30(1.3 \%)$ & $104(0.9 \%)$ & $1.43(0.95-2.15)$ & 0.087 \\
\hline \multicolumn{5}{|l|}{ Ethnicity } \\
\hline Jews & $59(1.6 \%)$ & $189(1.0 \%)$ & 1.54 (1.15-2.07) & 0.004 \\
\hline Arabs & $0(0.0 \%)$ & $1(0.1 \%)$ & NA & 0.658 \\
\hline
\end{tabular}

$O R$ odds ratio, $n$ number, $C I$ confidence interval

${ }^{\mathrm{a}}$ The prevalence of melanoma in cases when melanoma preceded BP (in cases) or preceded recruitment (in controls)

Bold: significant value

\section{The risk of developing melanoma among patients with bullous pemphigoid}

The incidence rate of melanoma was 1.42 (95\% CI $0.95-2.05)$ and 1.16 (95\% CI 0.96-1.37) per 1000 personyears among patients with BP and controls, respectively (Table 3). Compared to control subjects, the risk of incident melanoma was not significantly elevated among patients with BP, neither in the unadjusted (HR 1.18; 95\% CI $0.77-1.82 ; P=0.447$ ) nor in the adjusted (adjusted HR 1.13; 95\% CI $0.73-1.74 ; P=0.587$ ) analyses. This finding was not altered after a sensitivity analysis which included only BP patients managed by BP-related medications (Table 3).

\section{The time sequence of the appearance of the two investigated conditions}

Of the 85 patients with a dual diagnosis of BP and melanoma, BP followed melanoma in $59(69.4 \%)$ cases whilst it preceded melanoma in the remaining 26 (30.6\%) cases. When BP followed melanoma, the mean (SD) interval between the conditions was 9.2 (7.7) years, and the median

Table 3 Incidence rates and hazard ratio of new-onset melanoma among patients with bullous pemphigoid (cohort study design)

\begin{tabular}{|c|c|c|}
\hline & Patients with bullous pemphigoid & Controls \\
\hline Follow-up time, PY & $18,320.9$ & $106,465.8$ \\
\hline Median follow-up time, years (range) & $3.49(0.00-17.64)$ & $4.66(0.00-17.85)$ \\
\hline Number of events of melanoma & 26 & 123 \\
\hline Incidence rate/1000 PY & 1.42 & 1.16 \\
\hline \multirow[t]{2}{*}{$95 \% \mathrm{CI}$} & $0.95-2.05$ & $0.96-1.37$ \\
\hline & HR $(95 \%$ CI $)$ & $P$ value \\
\hline Crude & $1.18(0.77-1.82)$ & 0.447 \\
\hline Adjusted $^{\mathrm{a}}$ & $1.13(0.73-1.74)$ & 0.587 \\
\hline \multicolumn{3}{|l|}{ Sensitivity analysis ${ }^{\mathrm{b}}$} \\
\hline Crude & $1.07(0.69-1.66)$ & 0.768 \\
\hline Adjusted $^{\mathrm{a}}$ & $1.06(0.67-1.65)$ & 0.809 \\
\hline
\end{tabular}

$H R$ hazard ratio, $C I$ confidence interval, $P Y$ person-year

${ }^{\mathrm{a}}$ Following the adjustment for age, sex, ethnicity, socioeconomic status, comorbidities, PD-1/PDL-1 exposure, healthcare utilization, and Parkinson diseases

bensitivity analysis included only bullous pemphigoid patients under prolonged "bullous pemphigoid-related treatments"

Bold: significant value 
(range) interval was 7.8 (0.1-36.0) years. When BP preceded the onset of melanoma, the average (SD) and the median (range) intervals were 3.5 (2.6) and $2.9(0.2-8.8)$ years, respectively.

\section{The epidemiological and clinical features of patients with BP and melanoma as compared to the remaining patients with $\mathrm{BP}$}

Patients with coexistent BP and melanoma were significantly older at the onset of BP (80.4[10.2] vs. 76.6[14.4]; $P=0.001$ ), had lower body mass index (25.8 [3.5] vs. 27.9 [6.1]; $P<0.001$ ), and higher exposure to PD-1/PDL-1 inhibitors $(1.2 \%$ vs. $0.1 \% ; P=0.004)$.

The two subgroups were comparable with respect to sex, ethnicity, Charlson comorbidity index, exposure to longterm systemic corticosteroids and adjuvant therapies, and the prevalence of smoking and the recently reported dipeptidyl peptidase 4 inhibitors (DPP4i)-associated BP (Table 4). The risk of all-cause mortality was comparable between patients with BP and comorbid melanoma as compared to those with isolated BP (HR 0.88; 95\% CI 0.65-1.20; $P=0.427$; Fig. 1).

\section{Discussion}

The current large-scale population-based study revealed that patients with a preexisting diagnosis of melanoma are at a 1.5-fold increased risk of developing BP. This association was most prominent among males and individuals older than

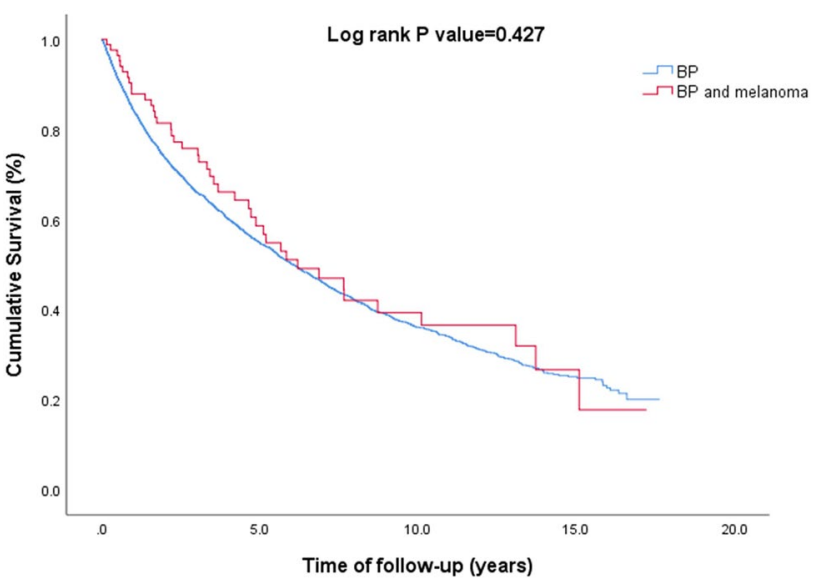

Fig. 1 A Kaplan-Meier curves demonstrating survival of patients with BP and comorbid melanoma relative to the remaining patients with BP

80 years of age and persisted following the adjustment for putative confounding factors, including exposure to PD-1/ PDL-1 antagonists. Compared to the remaining patients with $\mathrm{BP}$, those with $\mathrm{BP}$ and comorbid melanoma were older at the onset of BP and had lower BMI.

The association of BP with melanoma is poorly understood, as it is based merely on case reports. In 2017, Amber et al. [10] summarized the literature to reveal five cases of $\mathrm{BP}$ developing in association with melanoma. Intriguingly, the clinical course of BP paralleled that of melanoma in those reported patients, with several patients experiencing
Table 4 Comparison between patients with coexistent bullous pemphigoid and melanoma relative to the remaining patients with bullous pemphigoid

\begin{tabular}{lcrr}
\hline & $\begin{array}{l}\text { BP with } \\
\text { melanoma } \\
(n=85)\end{array}$ & $\begin{array}{l}\text { BP without } \\
\text { melanoma } \\
(n=3839)\end{array}$ & $P$ value \\
\hline Age at the onset of BP, years; mean (SD) & $80.4(10.2)$ & $76.6(14.4)$ & $\mathbf{0 . 0 0 1}$ \\
Male sex, $n(\%)$ & $44(51.8 \%)$ & $1623(42.3 \%)$ & 0.079 \\
Jewish ethnicity, $n(\%)$ & $84(98.8 \%)$ & $3668(95.5 \%)$ & 0.144 \\
Body mass index; kg/m ${ }^{2}$, mean (SD) & $25.8(3.5)$ & $27.9(6.1)$ & $<\mathbf{0 . 0 0 1}$ \\
Smoking, $n(\%)$ & $26(30.6 \%)$ & $1122(29.2 \%)$ & 0.779 \\
Charlson comorbidity Index (w/o malignancies); mean (SD) & $2.7(1.8)$ & $2.9(2.2)$ & 0.413 \\
Long-term systemic corticosteroids, $n(\%)^{\mathrm{a}}$ & $58(68.2 \%)$ & $2494(65.0 \%)$ & 0.540 \\
Long-term topical corticosteroids, $n(\%)^{\mathrm{b}}$ & $83(97.6 \%)$ & $3609(94.0 \%)$ & 0.164 \\
Adjuvant immunosuppressant or immunomodulatory agents ${ }^{\mathrm{c}}$ & $54(63.5 \%)$ & $2283(59.5 \%)$ & 0.457 \\
DPP4i-associated BP, $n(\%)$ & $5(5.9 \%)$ & $291(7.6 \%)$ & 0.558 \\
PD-1/PDL-1 antagonists-associated BP, $n(\%)$ & $1(1.2 \%)$ & $5(0.1 \%)$ & $\mathbf{0 . 0 0 4}$ \\
\hline
\end{tabular}

$n$ number, $S D$ standard deviation, $w / o$ without, $D P P 4 i$ dipeptidyl peptidase-4 inhibitor, $P D$ programmed death, $P D L$ programmed death ligand

Bold: significant values

${ }^{\text {a}}$ Patients managed by systemic corticosteroids for more than 6 months

${ }^{b}$ Patients managed by topical corticosteroids for more than 6 months

${ }^{c}$ Patients managed by one of the following agents: azathioprine, mycophenolate mofetil, methotrexate, cyclophosphamide, dapsone, doxycycline, rituximab, plasmapheresis, intravenous immunoglobulins 
flares in BP in association with the development of metastatic diseases [19-21]. Correspondingly, patients with BP achieved a notable improvement in their cutaneous disease following the resection of the initial melanoma or the metastatic lymph nodes [10, 19].

A growing body of evidence accumulated in the past few years to suggest an association between administrations of PD-1/PDL-1 antagonists, part of which utilized to manage patients with metastatic melanoma, and the development of subsequent BP [16]. PD-1 and PDL-1 normally function as immune checkpoints which prevent the activation of T-cells and promote self-tolerance. The PD-1/PD-L1 pathway represents an adaptive immune resistance mechanism that is utilized by tumors to escape immune surveillance [22]. PD-1/ PDL-1 directed monoclonal antibodies were recently developed and demonstrated promising anti-cancer activity [23], but were strongly associated with immune-related adverse events, including the induction of BP, which was proved morphologically and immunopathologically indistinguishable from typical BP [16]. Of great interest, the increased risk of BP among individuals with a history of melanoma persisted following the adjustment for these agents. This indicates that melanoma embodies an independent risk factor of BP regardless of exposure to PD-1/PDL-1 antagonists. It cannot be thoroughly refuted that the increased incidence of BP under PD-1/PDL-1 antagonists among metastatic melanoma patients actually represents an exacerbation of the underlying association between melanoma and BP.

The pathomechanism underlying the association between $\mathrm{BP}$ and melanoma is yet to be fully elucidated. However, several observations may lend weight to this epidemiological comorbidity. Krenacs et al. [11] found that the cell-residual $60 \mathrm{kDa}$ endodomain of BP180 is expressed in malignant melanoma but not in benign melanocytic tumors, namely common, blue, and Spitz nevi. The authors postulated that the accumulation of BP180 endodomain in melanocytic tumors is associated with malignant transformation. In their recent study, Hwan et al. [12] generated a mouse strain with BP180 dysfunction and showed that it is associated with myeloidderived suppressor cells (MDSC) influx into the skin as well as with melanoma progression. This study implies that BP180 exerts an antitumor effect through modulating the infiltration of MDSC. In a Japanese study, levels of anti-BP230 autoantibodies were significantly increased among 55 patients with melanoma relative to 27 healthy controls. Intriguingly, these autoantibodies were detected in melanoma patients at both early and advanced stages of disease [13]. A subsequent study comparing 179 patients with melanoma and 22 controls failed to find any significant differences, neither in seropositivity nor in levels of these autoantibodies [24]. The aforementioned studies throw light on a putative link between melanoma and the autoantigens of BP, which may contribute to understanding our epidemiological findings. Further research is required to better elucidate the mechanism of this comorbidity.

On the genetic level, human leukocyte antigen (HLA) polymorphisms might impose a predisposition for both $\mathrm{BP}$ and melanoma. HLA-DQB1*03:01 was found overrepresented in Caucasian patients with melanoma and independently predicted recurrence and metastasis [25, 26]. Likewise, HLADQB1*03:01 strongly interacts with BP180 and is the most established HLA allele to associate with BP [27, 28].

The current study represents the first population-based study aiming to investigate the association between melanoma and BP. Owing to the knowledge of the temporal sequence in which the diagnosis appeared, we were able to elucidate the risk of melanoma among patients with BP and the risk of subsequent BP in those with a history of melanoma [29]. The population-based setting enabled to include patients managed in all levels of healthcare facilities, thus arguing against the presence of selection bias. Being of a large-scale nature, the current study provides findings that are less likely to pop up due to chance. Since it is based on a computerized dataset, the current study lacked morphological and pathological data of the eligible patients (e.g., the stage of melanoma and severity of BP).

In conclusion, the current population-based study depicts that a history of melanoma places the patient at a $50 \%$ increased risk of developing subsequent melanoma. This risk was independent of the exposure to PD-1/PDL-1 antagonists and was robust to sensitivity and multivariate analyses. The current study provides the first epidemiological evidence of an association between BP and melanoma and substantiates previous scattered observations attributing a role for $\mathrm{BP}$ autoantigens in carcinogenesis of melanoma. Further experimental research is necessary to better delineate the mechanism underlying this association. Knowledge about this association may motivate physicians managing melanoma patents to avoid additional well-recognized risk factors of BP like exposure to DPP4i and neuropsychiatric medications.

Supplementary Information The online version contains supplementary material available at https://doi.org/10.1007/s00403-021-02211-4.

Funding Open Access funding enabled and organized by Projekt DEAL.

\section{Declarations}

Conflict of interest ADC served as an advisor, investigator, or speaker for Abbvie, BI, Dexcel Pharma, Janssen, Novartis, Perrigo, Pfizer, and Rafa. None of the other authors have any conflicts of interest to declare.

Open Access This article is licensed under a Creative Commons Attribution 4.0 International License, which permits use, sharing, adaptation, distribution and reproduction in any medium or format, as long as you give appropriate credit to the original author(s) and the source, provide a link to the Creative Commons licence, and indicate if changes 
were made. The images or other third party material in this article are included in the article's Creative Commons licence, unless indicated otherwise in a credit line to the material. If material is not included in the article's Creative Commons licence and your intended use is not permitted by statutory regulation or exceeds the permitted use, you will need to obtain permission directly from the copyright holder. To view a copy of this licence, visit http://creativecommons.org/licenses/by/4.0/.

\section{References}

1. Kridin K (2018) Subepidermal autoimmune bullous diseases: overview, epidemiology, and associations. Immunol Res [Internet]. 2018 Feb 21 [cited 2018 Apr 2] 66(1):6-17. http://www.ncbi. nlm.nih.gov/pubmed/29159697

2. Sawamura D, Li K, Chu ML, Uitto J (1991) Human bullous pemphigoid antigen (BPAG1): amino acid sequences deduced from cloned cDNAs predict biologically important peptide segments and protein domains. J Biol Chem 266(27):17784-17790

3. Muramatsu K, Ujiie H, Kobayashi I, Nishie W, Izumi K, Ito T et al (2018) Regulatory T-cell dysfunction induces autoantibodies to bullous pemphigoid antigens in mice and human subjects. J Allergy Clin Immunol [Internet]. 2018 Apr 26 [cited 2018 May 28]; http://www.ncbi.nlm.nih.gov/pubmed/29704593

4. Amber KT, Murrell DF, Schmidt E, Joly P, Borradori L (2018) Autoimmune subepidermal bullous diseases of the skin and mucosae: clinical features, diagnosis, and management. Clin Rev Allergy Immunol. 54:26-51

5. Kridin K, Ludwig RJ (2018) The growing incidence of Bullous pemphigoid: overview and potential explanations. Front Med [Internet]. 2018 Aug 20 [cited 2018 Sep 11];5(AUG):220. http:// www.ncbi.nlm.nih.gov/pubmed/30177969

6. Kridin K, Ludwig RJ, Schonmann Y, Damiani G, Cohen AD (2020) The bidirectional association between bullous pemphigoid and psoriasis: a population-based cohort study. Front Med 7:511. https://doi.org/10.3389/fmed.2020.00511

7. Schadendorf D, van Akkooi ACJ, Berking C, Griewank KG, Gutzmer R, Hauschild A et al (2018) Melanoma. Lancet 392:971-984

8. Conic RRZ, Ko J, Damiani G, Funchain P, Knackstedt T, Vij A et al (2019) Predictors of sentinel lymph node positivity in thin melanoma using the National Cancer Database. J Am Acad Dermatol. 80:441-447

9. MacKie RM, Hauschild A, Eggermont AMM (2009) Epidemiology of invasive cutaneous melanoma. Ann Oncol. https://doi. org/10.1093/annonc/mdp252

10. Amber KT, Panganiban CM, Korta D, De Feraudy S, Kelly KM, Grando SA (2017) A case report of bullous pemphigoid associated with a melanoma and review of the literature. Melanoma Res 27:65-67

11. Krenacs T, Kiszner G, Stelkovics E, Balla P, Teleki I, Nemeth I et al (2012) Collagen XVII is expressed in malignant but not in benign melanocytic tumors and it can mediate antibody induced melanoma apoptosis. Histochem Cell Biol 138(4):653-667

12. Hwang BJ, Zhang Y, Brozowski JM, Liu Z, Burette S, Lough $\mathrm{K}$ et al (2019) The dysfunction of BP180/collagen XVII in keratinocytes promotes melanoma progression. Oncogene 38(50):7491-7503

13. Shimbo T, Tanemura A, Yamazaki T, Tamai K, Katayama I, Kaneda Y (2010) Serum anti-BPAG1 auto-antibody is a novel marker for human melanoma. PLoS ONE 5(5):e10566

14. Greenland S, Thomas DC (1982) On the need for the rare disease assumption in case-control studies. Am J Epidemiol. 116:547-553
15. Cohen AD, Dreiher J, Regev-Rosenberg S, Yakovson O, Lieberman N, Goldfracht M et al (2010) The quality indigators program in Clalit Health Services: the first decade. Harefuah 149:204-209

16. Lopez AT, Khanna T, Antonov N, Audrey-Bayan C, Geskin L (2018) A review of bullous pemphigoid associated with PD-1 and PD-L1 inhibitors. Int J Dermatol [Internet]. 2018 Jun [cited 2018 May 29];57(6):664-9. http://www.ncbi.nlm.nih.gov/pubme $\mathrm{d} / 29630716$

17. Lai Y, Yew Y, Lambert W (2016) Bullous pemphigoid and its association with neurological diseases: a systematic review and meta-analysis. J Eur Acad Dermatol Venereol [Internet] 30(12):2007-2015. https://doi.org/10.1111/jdv.13660

18. Huang P, Yang XD, Di Chen S, Xiao Q (2015) The association between Parkinson's disease and melanoma: a systematic review and meta-analysis. Translational Neurodegeneration 4:21. https:// doi.org/10.1186/s40035-015-0044-y

19. Marks JM, Gold SC (1961) Pemphigoid with malignant melanoma. J R Soc Med 54(3):225-226

20. Parsons RL, Savin JA (1968) Pemphigoid and malignancy. Br J Cancer 22(4):669-672

21. Beck KM, Dong J, Geskin LJ, Beltrani VP, Phelps RG, Carvajal $\mathrm{RD}$ et al (2016) Disease stabilization with pembrolizumab for metastatic acral melanoma in the setting of autoimmune bullous pemphigoid. J Immunother Cancer 4(1):1-5

22. Shaabani S, Huizinga HPS, Butera R, Kouchi A, Guzik K, Magiera-Mularz K et al (2018) A patent review on PD-1/PD-L1 antagonists: small molecules, peptides, and macrocycles (2015-2018). Expert Opin Ther Patents 28:665-678

23. Chen Y, Pei Y, Luo J, Huang Z, Yu J, Meng X (2020) Looking for the optimal PD-1/PD-L1 inhibitor in cancer treatment: a comparison in basic structure, function, and clinical practice. Front Immunol 11:1088. https://doi.org/10.3389/fimmu.2020.01088

24. Gambichler T, Scheitz R, Höxtermann S (2016) Serum antiBPAG1 autoantibody level is not a useful biomarker for cutaneous melanoma. Melanoma Res 26:88-89

25. Lu M, Thompson WA, Lawlor DA, Reveille JD, Lee JE (1996) Rapid direct determination of HLA-DQB1 * 0301 in the whole blood of normal individuals and cancer patients by specific polymerase chain reaction amplification. J Immunol Methods 199(1):61-68

26. Bateman AC, Turner SJ, Theaker JM, Howell WM (1998) HLADQB $1 * 0303$ and $* 0301$ alleles influence susceptibility to and prognosis in cutaneous malignant melanoma in the British Caucasian population. Tissue Antigens 52(1):67-73

27. Büdinger L, Borradori L, Yee C, Eming R, Ferencik S, GrosseWilde $\mathrm{H}$ et al (1998) Identification and characterization of autoreactive $T$ cell responses to bullous pemphigoid antigen 2 in patients and healthy controls. J Clin Investig 102(12):2082-2089

28. Amber KT, Zikry J, Hertl M (2017) A multi-hit hypothesis of bullous pemphigoid and associated neurological disease: is HLADQB $1 * 03: 01$, a potential link between immune privileged antigen exposure and epitope spreading? Hla 89:127-134

29. Höfler M (2005) The Bradford Hill considerations on causality: a counterfactual perspective. Emerg Themes Epidemiol [Internet] 2:11. http://www.pubmedcentral.nih.gov/articlerender.fcgi?artid $=1291382 \&$ tool $=$ pmcentrez\&rendertype $=$ abstract

Publisher's Note Springer Nature remains neutral with regard to jurisdictional claims in published maps and institutional affiliations. 